\title{
FID Fast Image Display for (.BMP \& .PCX) Images
}

Rawaa Qasha

rawa_qasha@uomosul.edu.iq
Ahmed S. Nori

ahmed.s.nori@uomosul.edu.iq

Dept. of Computer Science

College of Computer Science and Mathematics

University of Mosul

Received on: 10/04/2002

Accepted on: 12/10/2003

\section{ABSTRACT}

Video display speed constitutes a very important factor in modern software performance. The best way to achieve fastest display is by accessing Video RAM and programming video card directly. In addition to the speed, this method provides flexibility and high performance video display operations. Besides that, dealing with $64 \mathrm{~K}$ and $16.7 \mathrm{M}$ color mode can be achieved only by this method.

Fast Image Display (FID) software is developed to display two popular types of images (BMP, PCX) using direct access to VRAM method with various SVGA modes differing in resolutions and number of colors. Assembly instructions and $\mathrm{C}++$ language have been used to write Software parts.

Keywords: VRAM, BMP, PCX, SVGA.

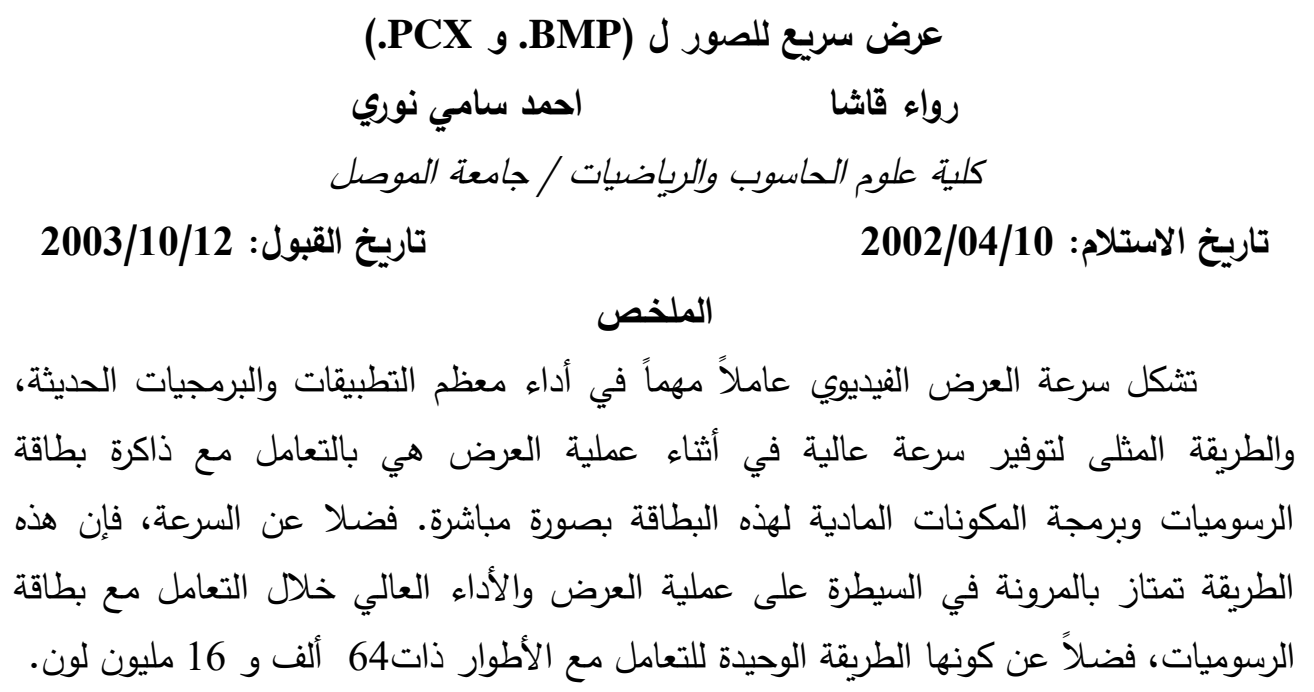




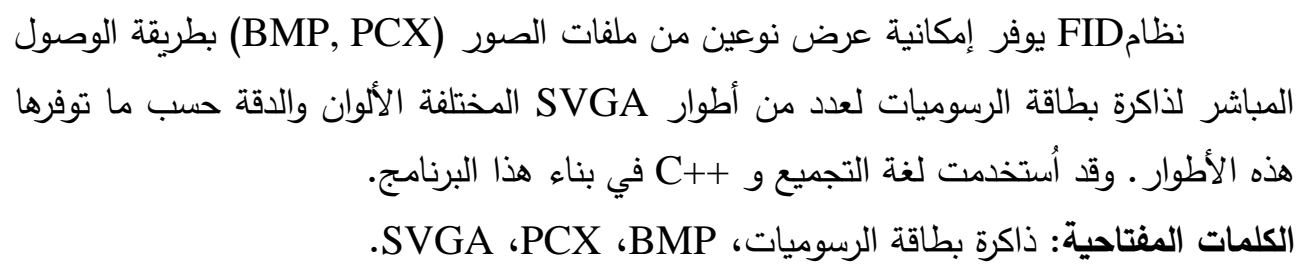

\section{Introduction}

As a general rule, the video display is created using either DOS or BIOS services that appropriate to the task in hand.

DOS and BIOS provide several functions for display manipulation, these functions are slow because the mechanism used to call these functions is slow and call BIOS interrupt takes much longer than a routine within a program. [1][7]

Besides that, BIOS supports capabilities to deal with at most 256 color modes, i.e graphics modes with more than 256 color cannot be managed by BIOS functions. While all modern video cards support graphics modes with high and true color with high resolution and required fast manipulation for graphics display. Direct manipulating video system offers fast and professional mechanism for graphics display and provides tools to deal with high color $(64 \mathrm{~K}$ color) and true color $(16.7 \mathrm{M}$ color) graphics modes.

\section{Direct Access Display method}

Each video card contains an appropriate amount of memory called Video RAM (VRAM) that stores information related to each pixel appears on the screen. Direct access technique 
accesses pixel's address in VRAM to change its information, this address can be calculated by means of its coordinates on the screen. For all applications to work on all PC's, the addresses used by each system must be the same. Therefore, to make individual pixel visible on the screen, the program must calculate the exact address of this pixel and pass the related color value to that address. [2][6]

Address calculation for a pixel depends on the number of bits required to represent each pixel and the organization of VRAM, which differs in each graphics mode. This method begins by setting an appropriate mode and calculates pixel address, then change its color.

\section{Video RAM}

Video cards offer different graphics modes with different resolutions, and various numbers of colors depending on the size of VRAM supported by the cards.

A standard VGA card provides $256 \mathrm{~K}$ bytes of VRAM, while SVGA card and its extended modes requires more than this amount, because of the new graphics modes with high resolution up to $1600 \times 1200$ and Large color spectrum $(32 \mathrm{~K}, 64 \mathrm{~K}, 16 \mathrm{M}$ color). [8]

VRAM is connected with the video card and the microprocessor. Microprocessor accesses VRAM as it can with 
ordinary RAM, while display hardware takes values from this memory and displays them on the screen. [2][9]

The organization of VRAM varies as the graphics modes differ. In general, VRAM is accessed through segment A starting at A000:0000h when the video card operates in graphics modes, which requires only $64 \mathrm{~K}$ bytes since, only segment $\mathrm{A}$ is available to the video card from the total addressable memory of PC., that means, 256K bytes of VRAM in standard VGA must addressed in $64 \mathrm{~K}$ bytes area, so that VRAM is divided into four equal sections, each section is called a bitplanes. Note that, the four bitplanes are not occupying different address spaces in memory, instead, all four bitplanes occupy the same address space. They all start at address A000H. [1][3][11]

\section{Latch Registers}

Communication between the processor and VRAM is managed using four 8-bit registers known as the latch registers. There is one-latch register for each bitplane, see figure (1). [12]

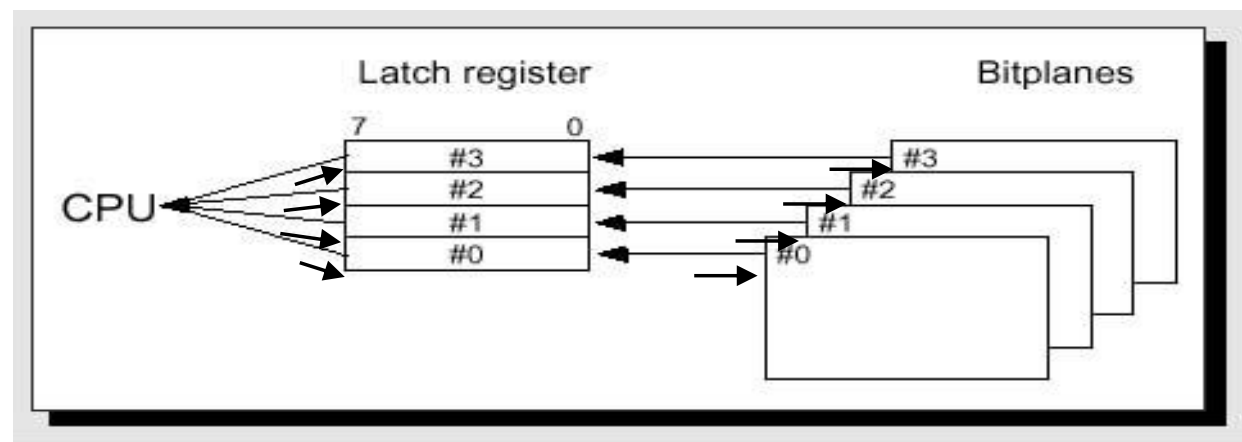

Figure (1): Bitplanes and Latch register 


\section{Organization of VRAM in 256 color modes}

In these modes, each pixel needs a byte in the VRAM to store its information in one of the four bitplanes. Thus all pixels are spreaded on the four bitplanes as follows:

Each four consecutive pixels information are found in the same offset address from the bitplanes. For example, offset address $(000 \mathrm{~h})$ in each bitplanes hold information related to the first four pixels in the screen starting at the upper left corner. First pixel in bitplane 0, seconds one in bitplane 1 and so on. The next four pixels are stored in offset address $0002 \mathrm{~h}$. In the same way, the remaining pixels are stored in the bitplanes as shown in figure (2):

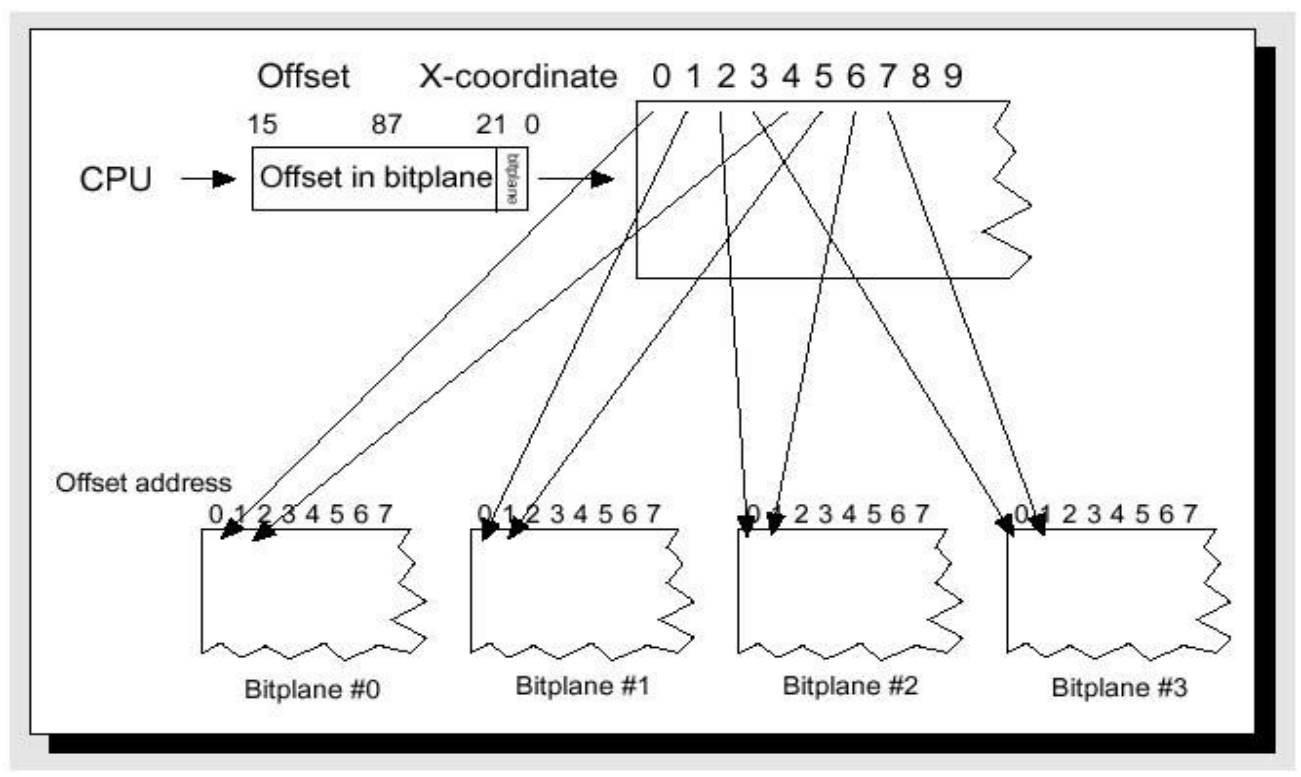

Figure (2): Alternate 256 color mode as seen by the CRTC 


\section{Accessing VRAM in 256-color mode}

According to the organization described in the previous section, each bitplane is addressed individually, so all pixels are not available in the $64 \mathrm{~K}$ byte at $\mathrm{A} 0000 \mathrm{~h}$ at the same time. Therefore accessing the address of a pixel with $(\mathrm{x}, \mathrm{y})$ as the horizontal and vertical coordinates respectively is calculated by multiplying y coordinates by (mode horizontal resolution /4), then the $\mathrm{X}$ coordinate is divided by 4 , since there are four pixels located at the same address. The sum of these two calculations is the address in one of the four bitplanes, see table (1), mathematically [3][5]:

Pixel address $(X, Y)=\mathrm{Y} \times($ mode horizontal resolution $/ 4)+\mathrm{X} / 4$

The number of the bitplane that holds the desired pixel must be loaded to one of the sequencer controller registers (Map Mask register). This number is obtained from the two lowest bits of the $\mathrm{X}$-coordinate.

Table (1): 256-color SVGA modes supported by FID software

\begin{tabular}{|c|c|c|c|c|}
\hline \multirow{2}{*}{ Mode No. } & \multicolumn{2}{|c|}{ Graphic Resolution } & No. of bits & Maximum \\
& Horizontal & Vertical & pixel & memory \\
\hline $101 \mathrm{H}$ & 640 & 480 & 8 & 307,200 \\
\hline $103 \mathrm{H}$ & 800 & 600 & 8 & 480,000 \\
\hline $105 \mathrm{H}$ & 1024 & 768 & 8 & 786,432 \\
\hline $107 \mathrm{H}$ & 1280 & 1024 & 8 & $1,310,720$ \\
\hline
\end{tabular}


The following equations are used to access any pixel in the modes described in table 1 :

- Offset $=\mathrm{Y} \times(160)+$ int $(\mathrm{X} / 4) \quad$ for mode $101 \mathrm{H}$

- Offset $=\mathrm{Y} \times(200)+\operatorname{int}(\mathrm{X} / 4) \quad$ for mode $103 \mathrm{H}$

- Offset $=\mathrm{Y} \times(256)+\operatorname{int}(\mathrm{X} / 4) \quad$ for mode $105 \mathrm{H}$

- Offset $=\mathrm{Y} \times(320)+\operatorname{int}(\mathrm{X} / 4) \quad$ for mode $107 \mathrm{H}$

\section{High color SVGA modes}

Each pixel in high color mode is represented by two bytes in VRAM. These two bytes are divided into three parts that corresponding to red, green, and blue intensities. the least five significant bits represents red intensity followed by six bits for green intensity, and five most significant bits for blue intensity. Organization of VRAM in $64 \mathrm{~K}$ high color mode is depicted figure (3)

Figure (3): Organizati on of VRAM in 64Khigh color mode.

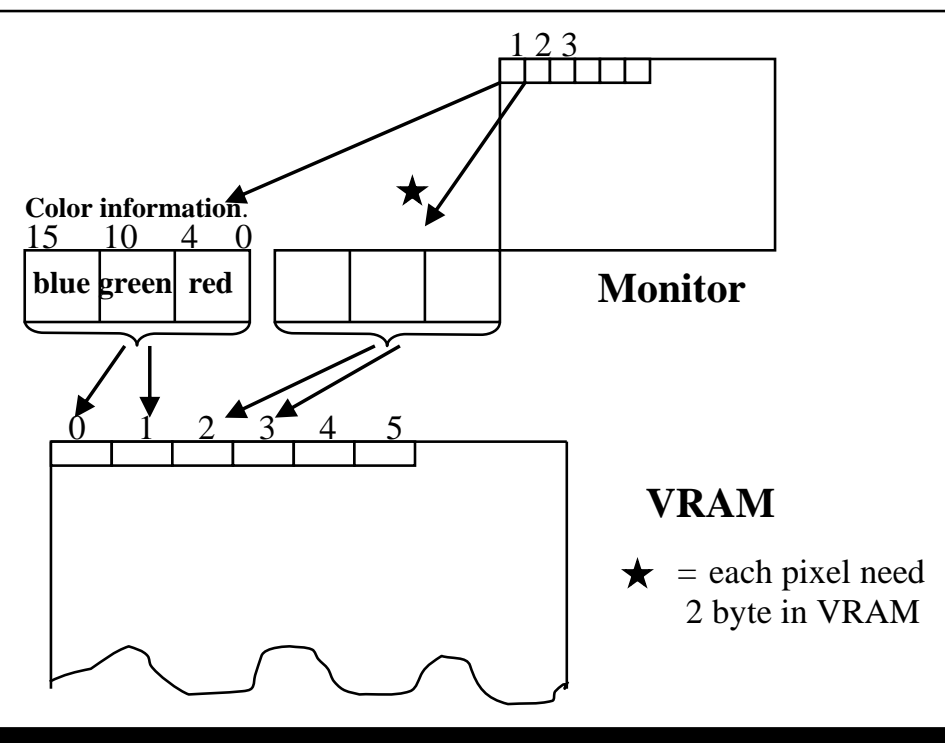




\section{Accessing VRAM in 64K-high color mode}

Pixel information in high color mode is managed in the $64 \mathrm{~KB}$ area in A segment. The two bytes that represent any pixel are existed in two consecutive address locations in the video memory, see table (2).

Address of the first byte for a pixel of $\mathrm{X}, \mathrm{Y}$ coordinate is calculated using the following equation:

Pixel-address $(\mathbf{X}, \mathbf{Y})=\mathrm{Y} \times($ mode horizontal resolution $\times 2)+\mathrm{X} \times 2$ Offset address for the second byte can be obtained by incrementing first address by one.

Table (2): High colors SVGA modes used by FID software

\begin{tabular}{|c|c|c|c|c||}
\hline \multirow{2}{*}{ Mode No. } & \multicolumn{2}{|c|}{ Graphic Resolution } & No. of bits & Maximum \\
\cline { 2 - 3 } & Horizontal & Vertical & /pixel & memory \\
\hline $10 \mathrm{EH}$ & 320 & 200 & 16 & $128 \mathrm{~KB}$ \\
\hline $111 \mathrm{H}$ & 640 & 480 & 16 & 614,400 \\
\hline $114 \mathrm{H}$ & 800 & 600 & 16 & 960,000 \\
\hline $117 \mathrm{H}$ & 1024 & 768 & 16 & $1,572,864$ \\
\hline $11 \mathrm{AH}$ & 1280 & 1024 & 16 & $2,621,440$ \\
\hline
\end{tabular}

To access any pixel in any of the five modes, the following equations are used for each mode respectively.

- Offset $=\mathrm{Y} \times(320 \times 2)+\mathrm{X} \times 2$ for mode $10 \mathrm{EH}$

- Offset $=\mathrm{Y} \times(640 \times 2)+\mathrm{X} \times 2$ for mode $111 \mathrm{H}$

- Offset $=\mathrm{Y} \times(800 \times 2)+\mathrm{X} \times 2$ for mode $114 \mathrm{H}$ 
- Offset $=\mathrm{Y} \times(1024 \times 2)+\mathrm{X} \times 2$ for mode $117 \mathrm{H}$

- Offset $=\mathrm{Y} \times(1280 \times 2)+\mathrm{X} \times 2$ for mode $11 \mathrm{AH}$

\section{Organization of VRAM in True color modes}

Depending on SVGA card system, pixels are represented by different no. of bytes,

(a) 24-bit true color mode assign 3 bytes for each pixel, one for red intensity, one for green intensity and other for blue intensity, as depicted

Figure (4):

Organizati on of VRAM in 24-True color mode.

(b) in figure

(c) (4). 
(d) 32-bit true color assigns 4 bytes for each pixel, three for red, green, blue intensities, and the last byte is added

Figure (5): Organization of VRAM in 32-True color mode.

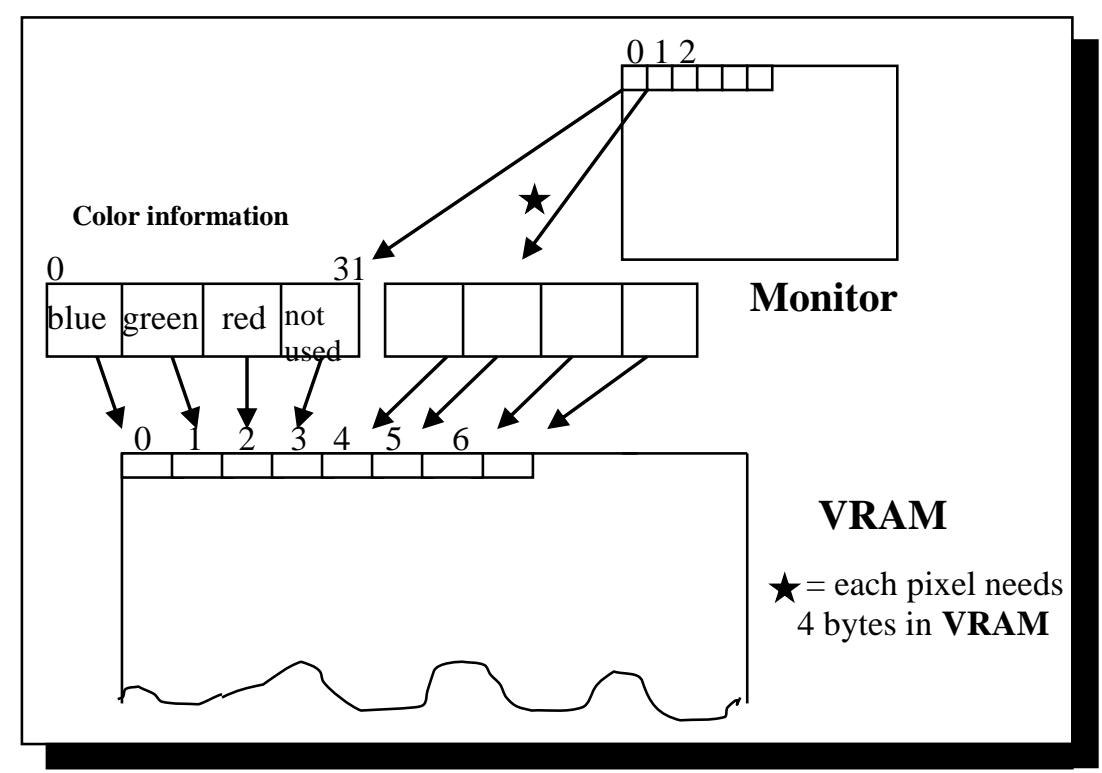

for future using, figure (5) depicts the organization of VRAM in 32-true color mode.

\section{Accessing VRAM in True color modes}

Here, there is no need for bitplanes, instead the information is managed in the $64 \mathrm{~K}$ byte area in segment $\mathrm{A}$.

If the video card support 24-bit true color, then the three bytes for each pixel are founded in three consecutive addresses in VRAM. So to change color information for any pixel with $X \& Y$ coordinates, calculate its first byte offset address by multiplying Y coordinate by scanline width (mode horizontal resolution $\times 3$ ), and multiply the $\mathrm{X}$-coordinate by 3 . Then the sum of these two 
calculations can be used to access the first byte, see table (3), mathematically [3][4][10]:

Pixel Offset $(X, Y)=\mathrm{Y} \times($ mode horizontal resolution $\times 3)+\mathrm{X} \times 3$ The offset address for the other two bytes is obtained by incrementing the first address.

Also in 32-bit true color mode, the four bytes that representing any pixel are found in four consecutive addresses and the offset address for the first byte is calculated using the following equation:

Pixel Offset $(X, Y)=Y \times($ mode horizontal resolution $\times 4)+X \times 4$

This offset address is incremented to obtain the other three bytes.

Table (3): True colors SVGA modes accessed by FID software

\begin{tabular}{|c|c|c|c|c|}
\hline \multirow{2}{*}{ Mode No. } & \multicolumn{2}{|c|}{ Graphic Resolution } & No. of bits & Maximum \\
/pixel & Horizontal & Vertical & 32 & $256 \mathrm{~KB}$ \\
\hline $10 \mathrm{FH}$ & 320 & 200 & 32 & $512 \mathrm{~KB}$ \\
\hline $10 \mathrm{FH}$ & 320 & 400 & 32 & $1,228,800$ \\
\hline $112 \mathrm{H}$ & 640 & 480 & 32 & $1,920,000$ \\
\hline $115 \mathrm{H}$ & 800 & 600 & 32 & $3,145,728$ \\
\hline $118 \mathrm{H}$ & 1024 & 768 & \multicolumn{3}{|l}{} \\
\hline
\end{tabular}

To access any pixel in any of the four listed modes, the following equations are used for each mode respectively. 


\section{4-bit True color equations:}

- Offset $=\mathrm{Y} \times(320 \times 3)+\mathrm{X} \times 3 \quad$ for mode $10 \mathrm{FH}$

- Offset $=\mathrm{Y} \times(640 \times 3)+\mathrm{X} \times 3 \quad$ for mode $112 \mathrm{H}$

- Offset $=\mathrm{Y} \times(800 \times 3)+\mathrm{X} \times 3$ for mode $115 \mathrm{H}$

- Offset $=\mathrm{Y} \times(1024 \times 3)+\mathrm{X} \times 3 \quad$ for mode $118 \mathrm{H}$

\section{2-bit True color equations:}

- Offset $=\mathrm{Y} \times(320 \times 4)+\mathrm{X} \times 4 \quad$ for mode $10 \mathrm{FH}$

- Offset $=\mathrm{Y} \times(640 \times 4)+\mathrm{X} \times 4$ for mode $112 \mathrm{H}$

- Offset $=\mathrm{Y} \times(800 \times 4)+\mathrm{X} \times 4$ for mode $115 \mathrm{H}$

- Offset $=\mathrm{Y} \times(1024 \times 3)+\mathrm{X} \times 4$ for mode $118 \mathrm{H}$

\section{Accessing the extended VRAM in SVGA card}

As mentioned earlier, SVAG cards contain large amount of VRAM to store the whole screen pixels in high resolution and true color modes. These modes need more than $256 \mathrm{~KB}$ of VRAM, so accessing the extended VRAM is accomplished by mapping portions of the video memory into the $64 \mathrm{~K}$ window at A000h. So that VRAM is divided into pages, which corresponding to the granularity of $64 \mathrm{~K}$ window.

Window granularity is defined as the number of bytes between the closest two bytes in VRAM that can be placed on any address in the $64 \mathrm{~K}$ window, it can take $1 \mathrm{~K}, 2 \mathrm{~K}, 4 \mathrm{~K} \ldots$ etc. To determine the granularity allowed by SVGA systems, VESA 
BIOS function $(01 \mathrm{H})$ must be used, which returns information about a specific SVGA mode in a block structure.

\section{Comparison (DOS, BIOS and Direct Access)}

Its too difficult to decide with a specific manner the speed of video display by using each of (DOS \& BIOS) services and the Direct Access way, because the results depends on the programming details and the system hardware components. But, as an average, the speed ratio of Direct Access to DOS is about 20:1 and to BIOS is about 10:1.

Besides, for the graphics display, BIOS provides dealing with graphics modes up to 256 colors, but for the (true \& high) color graphics modes they can't be accessed only through the Direct Access way.

\section{Conclusion}

Direct access display is the fastest way for video display that supports powerful abilities to manipulate the extended memory associated with the video modes of SVGA cards by controlling the operations of video mode initialization and VRAM access.

These operations performed by writing directly to VRAM, manipulating video card controllers registers and directly programming the Palette and DAC registers. Direct access 
abilities make it possible to deal with high and True color modes (24-bit, 32-bit) in high resolutions.

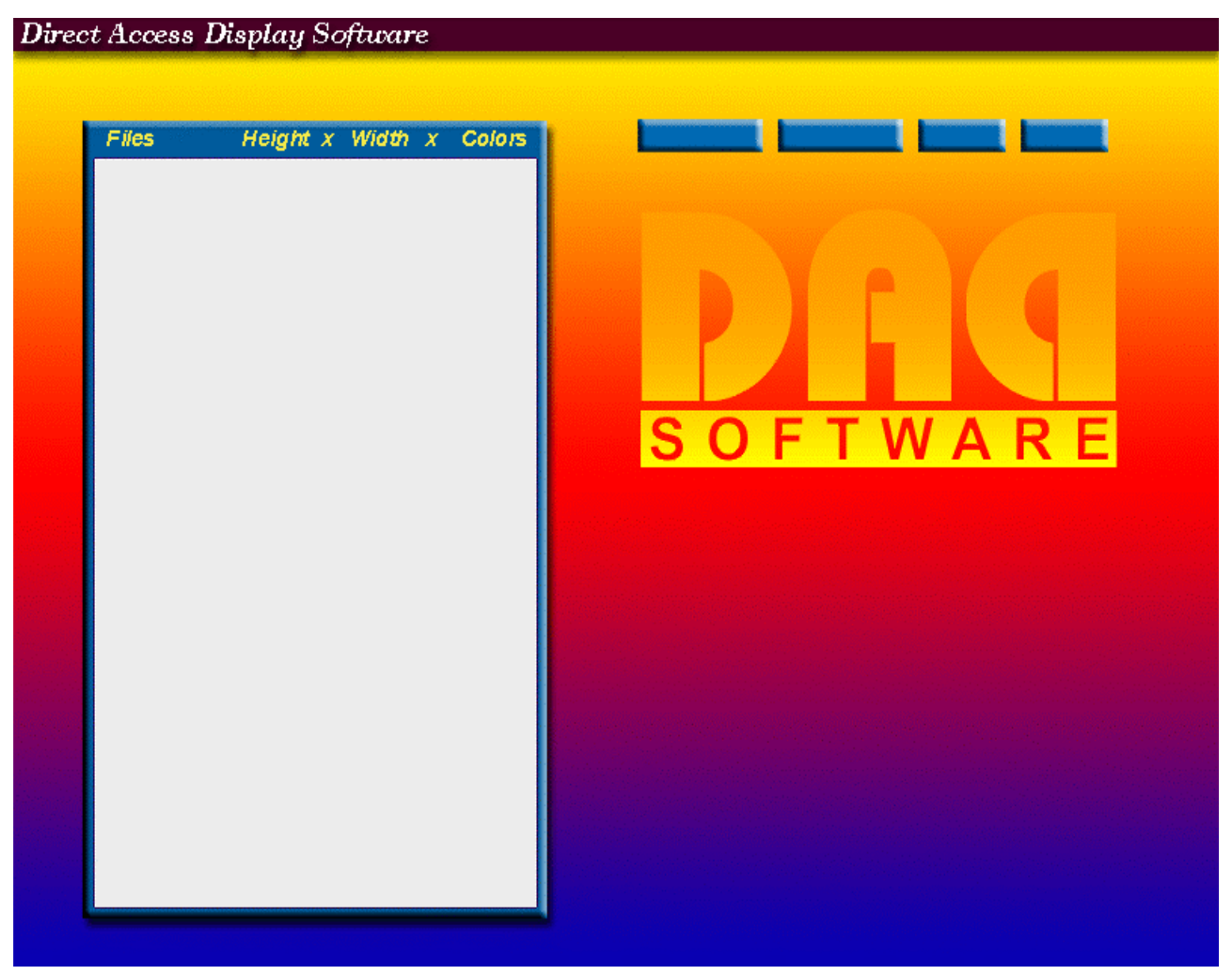

\section{REFERENCES}

1. Deltenei, Justin, 2000, Inverse Reality, http:// www.inversereality.org/tutorials/graphics\%20program ming/graphics programming.html

2. Deltenei, Justin, 2000, Inverse Reality, http:// www.inversereality.org/tutorials/graphics\%20program ming/plottingpixelsVRAM.html

3. Introduction to VESA Programming, 
www.monstersoft.com/tutorial/1/vesa-intro.html

4. Jones, Chris, 1998, DOS Games Programming, http://

saturn.spaceports.com/ dosuser/dosgames.htm\#programmi ng\%20SVGA.

5. Lafore, R., 1994, "Turbo C Programming for the PC", The Waite Group Inc.

6. Norton, P., 1986, "Inside the IBM PC", Brady Books. Inc.

7. Phoenix Technologies Ltd., 1989, "System BIOS for IBM PC/ XT/ AT Computers and Compatibles", Addison-Wesley

8. Rosch, L. W., 1997, "Hardware Bible”, Premier Edition, Techmedia.

9. Sandler, C., 1998, "Fix Your Own PC", $4^{\text {th }}$ Edition, M.I.S. Press.

10. Tischer, M. \& Jennrich, B., 1996, "PC Intern", $6^{\text {th }}$ Edition, Abacus.

11. Wilton, R., 1987, “Programmer's Guide to PC and PS/2", Microsoft Press.

12. Wood, W., June 1989, "The PC Graphics Maze", Electronics and wireless World, V. 6, N. 4. 


\section{Appendix -A-}

\section{FID software Review}

The objective behind designing this software is to reach the high-speed display achieved by accessing VRAM directly through the displaying of several types of images, and provides an essential information about video display environment. FID offers the following capabilities:

1- Fast displaying BMP and PCX images with their information including image high and width, and its number of colors.

2- Four high-resolution modes to display 256 color image, five high color modes to display high color images and five true color modes to display true color images with various resolutions.

3- Displaying information when any mode has been selected includes:

- Mode resolution.

- Granularity of the VRAM window.

- Number of bytes required by each pixel line in VRAM.

- Number of bits per screen pixel.

4- Displaying information about the current VESA card and its capabilities such as:

- Card signature 
- VESA version

- Card manufacturer name.

- Listing the supported card modes.

The following keys can be used to interact with the interface:

Alt + Drive letter to change to any drive.

M to display Mode info.

C to display Card info.

$\mathrm{H} \quad$ to display Help list.

A to display About list.

up, down arrow to select image file $\&$ mode.

ESC to Exit the software.

\section{Software main functions}

FID software uses the following main functions to perform the essential operations to access VRAM and program different video card registers, which belongs to different controllers.

\section{-Get VESA mode information}

This function calls VESA function $01 \mathrm{H}$ to provide information about the selected VESA mode that will be stored in Mode structure.

Begin

Assigning VESA function $4 \mathrm{~F} 01 \mathrm{H}$ to $\mathrm{AX}$ register;

Load ES:DI registers with the address of Mode structure; (see appendix $B(2)$ )

Call INT 10H;

End. 


\section{Appendix -A-}

\section{-Get VESA card information}

One of the VESA functions is used in this function to provide information related to the current card.

Begin

Assigning VESA function $4 \mathrm{~F} 00 \mathrm{H}$ to $\mathrm{AX}$ register;

Load ES:DI registers with the address of VESA block structure that can be used to hold VESA card information; (see appendix $B(1)$ )

Call INT 10H;

End.

\section{-Set pixel with 256 color mode}

This function uses one of the equations described previously to access pixel address in 256 color modes depending on the selected mode.

Begin

Calculating offset address for pixel with X, Y coordinate If offset address exceed $64 \mathrm{~K}$ byte then

Call VESA function 4FH, subfunction $05 \mathrm{H}$ through INT 10H to access next VRAM window; 
Set Map Mask register in the sequencer controller to the content of least significant two bits of $\mathrm{X}$ coordinate to deal with proper bitplane;

Assign pixel color to its offset address;

End.

-Set pixel in high color mode

It's used to access pixel position in VRAM using any of the high color mode equation:

Begin

Calculating offset address using high color equation for the first pixel byte;

If offset address exceed $64 \mathrm{~K}$ Byte then

Call VESA subfunction $05 \mathrm{H}$ by using INT $10 \mathrm{H}$ to access next VRAM window

Assign a byte that contains five blue bits and green three most significant bits to the address

Increment address

Assign the remaining green bits with red bits to the second address

End.

-Set pixel in True color mode

Depending on true color mode type, this function uses either 32-bit true color equations or 24-bit equations to access pixel address. 
Begin

If selected mode is 32-bit mode then

Calculating offset address using 32-bit equation for the first pixel byte;

else

using 24-bit equation to calculate first pixel byte address;

If offset address exceed $64 \mathrm{~K}$ Byte then

Call VESA subfunction $05 \mathrm{H}$ by using INT $10 \mathrm{H}$ to access next VRAM window

Assigning the three colors values to three consecutive bytes within VRAM;

End.

\section{-Display BMP file using 256 color mode}

FID uses this function to read and display BMP file using the above functions.

Begin

Open BMP file

Reading file header information

Reading image header information

Reading RGB color array

Setting selected mode

Setting DAC registers to RGB array

Repeat

Reading byte color information from the file;

Call set pixel with 256-color function;

Until end of image file

End. 


\section{-Display BMP file with True color mode}

This function can be used to display BMP image in true color modes.

Begin

Open BMP file

Reading file header information

Reading image header information

Setting true color mode

Repeat

Read three bytes color information from the image file;

Calling set pixel with true color mode function;

Until end of file

End.

\section{-Display PCX file in 256 color mode}

FID uses this function to read, decode and display PCX image file.

\section{Begin}

Open PCX file

Reading file header information

Reading RGB array from the file

Setting 256 color mode

Setting DAC registers

Repeat

Reading one byte color information from the left;

Decoding color info

Calling set pixel function with 256 color;

Until end of image file 
End.

\section{Appendix -B-}

(1)

\begin{tabular}{|c|l|c|}
\hline \multicolumn{2}{|c|}{ SVGA mode structure } & \multicolumn{1}{|c|}{ Type } \\
\hline Ofs. & Contents & 1 word \\
\hline $00 \mathrm{H}$ & Mode flag, & 1 byte \\
\hline $02 \mathrm{H}$ & Flags for the first access window & 1 byte \\
\hline $03 \mathrm{H}$ & Flags for the second access window & 1 word \\
$04 \mathrm{H}$ & Granularity of the two access windows, in $\mathrm{K}$, & 1 word \\
\hline $06 \mathrm{H}$ & Size of the two access windows, in $\mathrm{K}$ & 1 word \\
\hline $08 \mathrm{H}$ & Segment address of the first access window & 1 word \\
\hline $\mathrm{OAH}$ & Segment address of the second access window & 1 dword \\
\hline $\mathrm{OCH}$ & FAR pointer to routine for setting the visible region in the two access windows & 1 byte \\
\hline $10 \mathrm{H}$ & Number of bytes required by each pixel line in video RAM word &
\end{tabular}

\section{VESA card information structure}

\begin{tabular}{|c|c|c|c|c|c|}
\hline Ofs. & Contents & Type & Ofs. & Contents & Type \\
\hline $\mathrm{OOH}$ & VESA Signature ("VESA") & 4BYTE & $\mathrm{O} 6 \mathrm{H}$ & $\begin{array}{l}\text { FAR pointer to an ASCII string } \\
\text { containing the name of the card } \\
\text { manufacturer }\end{array}$ & 1 DWORD \\
\hline $04 \mathrm{H}$ & $\begin{array}{l}\text { VESA Version, main version } \\
\text { number }\end{array}$ & 1BYTE & $\mathrm{OAH}$ & $\begin{array}{l}\text { Flag that indicates the capabilities of } \\
\text { the card currently not used, therefore } \\
0000 \text { h }\end{array}$ & 1 DWORD \\
\hline $05 \mathrm{H}$ & $\begin{array}{l}\text { VESA Version, secondary version } \\
\text { number }\end{array}$ & 1 BYTE & $\mathrm{OEH}$ & $\begin{array}{l}\text { FAR pointer to the list of code } \\
\text { numbers for the supported video } \\
\text { modes }\end{array}$ & 1 DWORD \\
\hline
\end{tabular}

